\title{
Study of Plasma Start-up Initiated by Second Harmonic Electron Cyclotron Resonance Heating on WEGA Experiment
}

\author{
M. Preynas ${ }^{\mathrm{a}}$, H.P. Laqua ${ }^{\mathrm{a}}$, M. Otte ${ }^{\mathrm{a}}$, T. Stange ${ }^{\mathrm{a}}$, T. Wauters ${ }^{\mathrm{b}}$, D. Aßmus ${ }^{\mathrm{a}}$ \\ ${ }^{a}$ Max Planck Institut für Plasmaphysik, EURATOM Association, D-17491 Greifswald, Germany \\ ${ }^{b}$ Association Euratom-Belgian State, LPP-ERM/KMS, 1000 Brussels, Belgium
}

\begin{abstract}
Although both $1^{\text {st }}$ harmonic ordinary mode $(\mathrm{O} 1)$ and $2^{\text {nd }}$ harmonic extra-ordinary mode (X2) have been successfully used to initiate pre-ionization and breakdown in many devices, a complete theoretical model is still missing to explain the success of this method. Moreover, some experimental observations are not completely understood, such as what occurs during the delay time between the turn-on of ECRH power and first signals of density or light measurements. Since during this free period the ECRH power has to be absorbed by in-vessel components, it is of prime importance to know what governs this delay time. Recently, dedicated start-up experiments have been performed on WEGA, using a $28 \mathrm{GHz}$ ECRH system in X2-mode. This machine has the interesting capability to be run also as a tokamak allowing comparative experiments between stellarator $(\iota / 2 \pi>0)$ and tokamak $(\iota / 2 \pi=0)$ configurations. Different scans in heating power, neutral gas pressure, and rotational transform $(l)$ show clearly that the start-up is a two step process. A first step following the turn-on of the ECRH power during which no measurable electron density (or just above the noise level in some cases), ECE and radiated power is detected. Its duration depends strongly on the level of injected power. The second step corresponds to the gas ionization and plasma expansion phase, with a velocity of density build-up and filling-up of the vessel volume depending mainly on pressure, gas and rotational transform. Moreover, an interesting scenario of ECRH pre-ionization without loop voltage in tokamak configuration by applying a small optimal vertical field can be relevant for start-up assistance on future experiments like ITER. The results from this experimental parametric study are useful for the modeling of the start-up assisted by the second harmonic electron cyclotron resonance heating. The aim of this work is to establish predictive scenarios for both ITER and W7-X operation.
\end{abstract}

Keywords: ECRH, Plasma Start-up, second extraordinary harmonic, stellarator, tokamak.

PACS: 52.35.-g, 52.35.Hr, 52.35.Qz, 52.55.Hc, 52.55.Fa

\section{INTRODUCTION}

One of the specific purposes and tasks of the Electron Cyclotron Resonance Heating (ECRH) system on both tokamaks and stellarators is to provide a reliable plasma start-up. Indeed, the plasma breakdown and burn-through has to be insured in the two upcoming biggest experiments in the world, ITER and W7-X, where large ECRH systems are foreseen: $24 \mathrm{MW}$ on ITER and $10 \mathrm{MW}$ on W7-X [1]. Contrary to the start-up in a tokamak where an induced loop voltage generates a plasma current, a reliable plasma start-up in a stellarator uses ECRH only (or NBI). Since the rotational transform (l) and thus the confining magnetic field already exist in vacuum, a loop voltage can be omitted. Therefore, the plasma start-up requires an external heating source in a stellarator, to compensate the lack of internal ohmic heating. In ITER, pre-ionization and assisted start-up with ECRH (or ICRH) will be necessary to achieve plasma breakdown because of the low toroidal electric field $(\leq 0.3 \mathrm{~V} / \mathrm{m})$ compared to the large volume of the vessel [2].

A typical delay time is observed between the ramp-up of the ECRH power and the electron density build-up. This delay time, also called breakdown time, can last up to several dozens of milliseconds. Since during this free period the ECRH power has to be absorbed by in-vessel components, it is of prime importance to know what governs the dependence of this delay time on discharge parameters (ECRH power characteristics, gas pressure and rotational transform) to avoid any damages during the operation of W7-X. Therefore, recent dedicated start-up experiments have been performed on the WEGA stellarator (IPP, Greifswald, Germany) [3], using a $10 \mathrm{~kW}-28 \mathrm{GHz}$ - ECRH system in X2-mode. WEGA has the interesting capability to be run also as a tokamak allowing comparative experiments between stellarator $(\iota / 2 \pi>0)$ and tokamak $(\iota / 2 \pi=0$, i.e. no poloidal field) configurations. Its major and minor radius are respectively $R=72 \mathrm{~cm}$ and $r=19 \mathrm{~cm}$. WEGA is currently operated with a magnetic field in the range of $[0.44-0.52] \mathrm{T}$, and a maximum rotational transform of about $\iota=0.4$ in stellarator configuration. The 
maximum plasma radius is $a=11 \mathrm{~cm}$ for a volume of $V=0.16 \mathrm{~m}^{3}$. A parametric study has been realized in both onand off-axis heating configuration in X2-mode. In the next part, the results from these experiments are summarized to give a characterization of the start-up on WEGA. Results from experiments of tokamak start-up configuration on WEGA are exposed in the third part of this paper. The last part is dedicated to the conclusion and perspectives.

\section{CHARACTERIZATION OF THE START-UP IN X2-MODE ON STELLARATOR}

Several parameters can influence the plasma start-up performance assisted by ECRH power. The first parameter is the level of power injected in the neutral gas (i.e. strength of the electric field), closely linked to the polarization and the harmonic of the electron cyclotron wave. The neutral gas pressure is a parameter to optimize, but each working gas has its own behavior in terms of plasma-wall interaction and plasma build-up. Results presented here correspond to helium and hydrogen discharges. Scans in ECRH power, neutral gas pressure and rotational transform are performed. To characterize the start-up, the delay time and the electron density $\left(n_{e}\right)$ of three series of helium discharges performed with on-axis heating are plotted as a function of the neutral gas pressure in figure 1. The delay time strongly depends on the pressure at low power $(2.8 \mathrm{~kW})$, since it increases from $18 \mathrm{~ms}$ to $60 \mathrm{~ms}$ when the pressure is increased from $5 \cdot 10^{-5}$ mbar to $25 \cdot 10^{-5}$ mbar (blue curve). However, no dependence on the pressure is observed at high power $(7 \mathrm{~kW})$, where the delay time remains constant at about $4 \mathrm{~ms}$ (dashed red curve). A small increase of $10 \%$ in the electron density is measured from the $5 \cdot 10^{-5}$ mbar discharge to the $25 \cdot 10^{-5}$ mbar discharge. The third discharge series (black dotted line) illustrates a high power case at very low rotational transform $(\iota / 2 \pi=$ 0.02). It shows that even at higher power $(8.3 \mathrm{~kW})$, the delay time is larger up a factor of 4 , and the electron density achieved during this discharge is especially much lower (factor of 1/4) than in high rotational transform discharges (red triangles $-\iota / 2 \pi=0.21$ ). These experiments show that there is an ECRH power threshold from which the startup performance does not vary over the scanned pressure range. However, this threshold depends on the rotational transform, which has to be strong enough to insure good confinement and thus high plasma density. Indeed, losses of high energy electrons (above the ionization energy threshold), which are accelerated by the EC wave-electron interaction, are minimized by a high rotational transform and an optimized neutral gas pressure. A too high neutral gas pressure inhibits the acceleration process by inelastic collisions between neutrals and electrons.
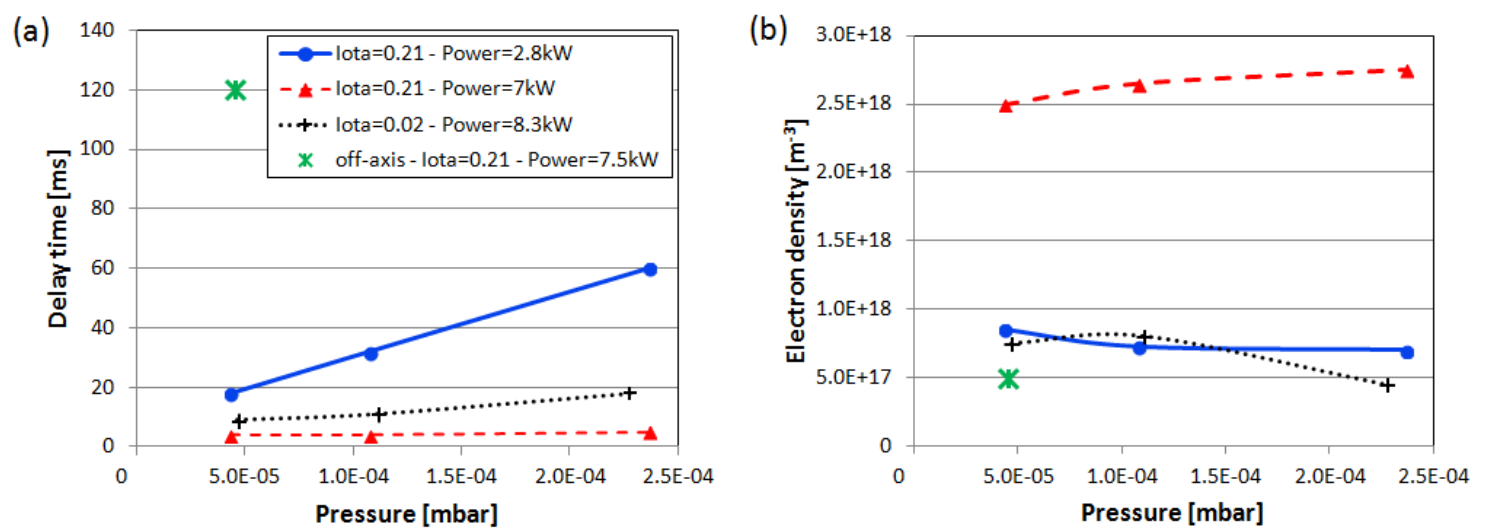

FIGURE 1. (a): Delay time (in ms) as a function of the neutral gas pressure (in mbar) for four different cases. The blue line corresponds to low ECRH power discharges ( $2.8 \mathrm{~kW}$ injected) at high rotational transform $(0.21)$. The dashed red curve represents discharges performed at the same iota but at higher power $(7 \mathrm{~kW})$. Then, a third discharge series at even higher power $(8.3 \mathrm{~kW})$ but very low rotational transform $(0.02)$ is illustrated by the dotted black curve. Finally, one off-axis discharge (green star) at high power $(7.5 \mathrm{~kW})$ and rotational transform $(0.21)$ is plotted. (b): Electron density as a function of the neutral gas pressure measured for the same sets of discharges.

Similar discharges have been performed in hydrogen gas to allow a comparison of the start-up performance between both gases. Results from high power ECRH discharges are plotted in figure 2, which shows that the start-up is faster in helium with an electron density up to a factor of 5 higher than in hydrogen. 

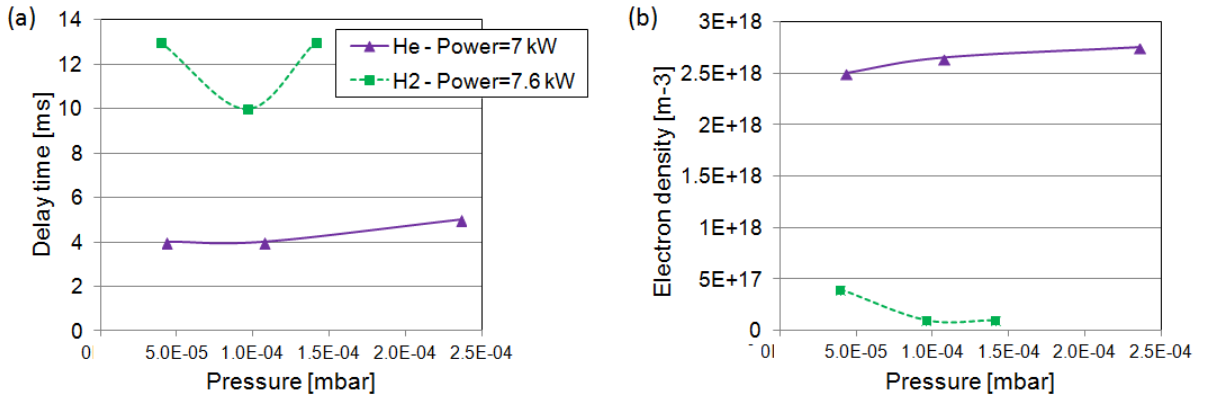

FIGURE 2. (a): Delay time as a function of the neutral gas pressure for helium and hydrogen plasma. (b): Electron density as a function of the neutral gas pressure measured for the same sets of discharges.

As previously observed on the Heliotron J helical device [4], only a thin ring of plasma is produced around the magnetic axis in a first phase. This phase, well reproduced on WEGA, especially during on-axis hydrogen discharges, is illustrated in figure 3-(a) by a picture taken with a video camera. It is characterized by a small increase in the electron density hardly measurable by the central channel of the interferometer, since only a thin volume around the magnetic axis is ionized (picture (a) in figure 1). Indeed, although the increment of energy by waveelectron interaction is low for the X2 scheme, a part of these electrons can accumulate energy by collisionless heating well above the energy of ionization of a neutral atom [5]. In a second phase, if good conditions are fulfilled in terms of ECRH power, neutral pressure and rotational transform, the radial plasma expansion phase occurs by perpendicular diffusion. The electron multiplication dominates the losses (drifts, recombination, etc), leading to a high increase in the electron density before it reaches a flat top (picture (b) in figure 3 ).
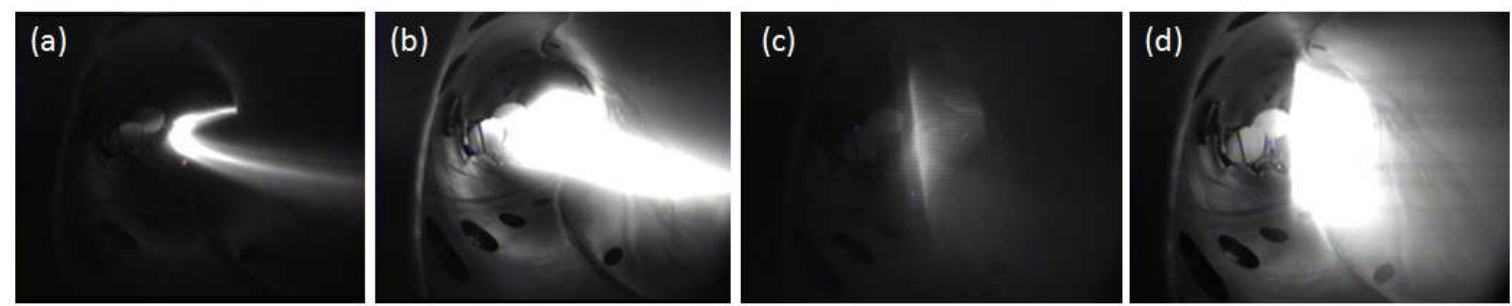

FIGURE 3. Illustration of the two main phases in the density build-up, in a stellarator configuration with rotational transform (pictures (a) and (b)), and in a tokamak configuration with vertical field (pictures (c) and (d)). (a): First, electron collisionless heating phase, when only a thin snake of plasma is produced around the magnetic axis. (b): Then, plasma expansion, associated to the electron density build-up. (c): Without the additional poloidal field, the confinement is poor and ionization occurs first in the vertical resonance cylinder. (d): optimized vertical field enables to ionize a large volume of neutral gas and to reach a high $\mathrm{n}_{\mathrm{e}}$.

Off-axis heating experiments were performed to complete the understanding of the electron density build-up. In this case, the EC resonance is shifted of about $6.5 \mathrm{~cm}$ away from the magnetic axis to the high field side, which is quite large compared to the radial size of the plasma $(\sim 11 \mathrm{~cm})$. A strong degradation in the plasma build-up is measured during the off-axis discharges. To illustrate this result, delay time and electron density from a discharge performed in optimized conditions (high iota and low pressure) is plotted in green marks in figure 1. This discharge can be compared with the low pressure discharge plotted with a red triangle since both are similar in terms of pressure and ECRH power injected. In off-axis heating, the delay time is much longer (120 ms) and the electron density is by a factor of 5 less. In this configuration, the ECRH beam does not cross the resonance on the magnetic axis leading to a less efficient process of electron acceleration by the EC wave.

\section{TOKAMAK SCENARIO FOR ECRH ASSISTED START-UP WITHOUT LOOP VOLTAGE}

In the discharges performed on WEGA in tokamak configuration, no poloidal field is applied resulting in a rotational transform equal to zero. In order to study the start-up assisted by ECRH and to evaluate its efficiency to pre-ionize the plasma, a constant vertical field is applied without action of the central solenoid. Indeed, thanks to the capability of WEGA to perform long pulses (20s), the ramp-up (or -down) of the vertical field ends $2.5 \mathrm{~s}$ before the 
ECRH power is switched-on to insure a zero loop voltage start-up. The line integrated electron density measured by the interferometer, as well as the delay time and the plasma current, is plotted as a function of the vertical field in figure 4 for a set of 12 discharges. The wide scan in vertical field shows that an optimal value of vertical field allows a fast plasma start-up. During these discharges, the increase in the electron density during the radial expansion phase of the plasma is synchronized with a plasma current driven by fast electrons generated by ECRH. Electrons are well confined by the compensation of the inward Lorentz force with the outward centrifugal force, and generate co- or counter-current depending on the vertical field sign. An absolute vertical field of [10-20] Gauss allows the confinement of electrons in the range of [150-220] keV. However, although best plasma start-up is achieved for an absolute value of vertical field of 20 Gauss (see pictures (c) and (d) in figure 3), an asymmetry in the results is observed between the positive and negative vertical field. Both electron density and plasma current are up to a factor of 3 higher in the case of negative vertical field. Contrary to a positive vertical field, a negative one is assumed to compensate the vertical $B \wedge \nabla \mathrm{B}$ drift by a vertical pitch angle.
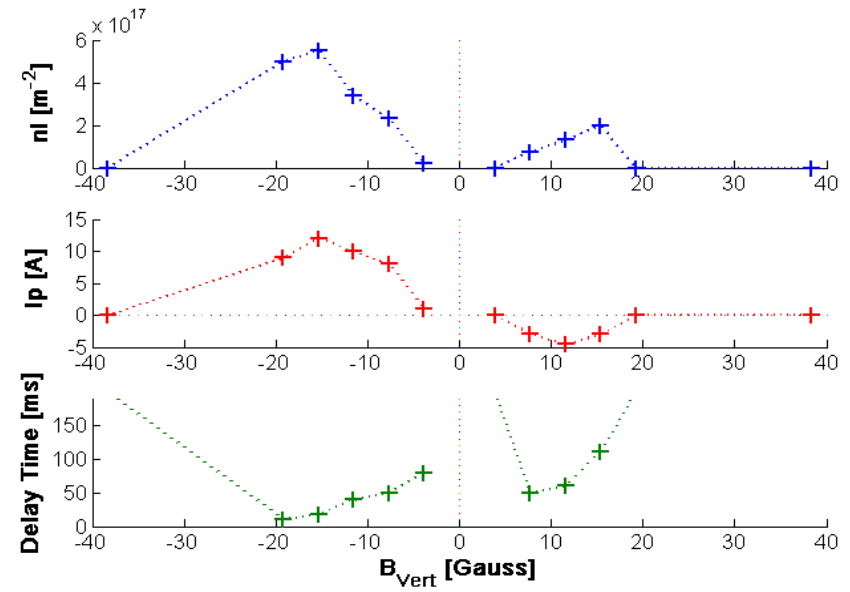

FIGURE 4. Line integrated electron density, plasma current and delay time as a function of the vertical field.

\section{CONCLUSION AND PERSPECTIVES}

Different scans in power, pressure, and rotational transform allowed the characterization of the plasma start-up on WEGA assisted by the ECRH in X2-mode. The optimized parameters, which were determined to achieve a fast start-up at high electron density, are the maximum ECRH power available on WEGA $(9 \mathrm{~kW})$, a low neutral pressure around $5.10^{-5} \mathrm{mbar}$, and on-axis EC resonance discharge in helium neutral gas.

The optimized scenario combining ECRH and vertical field to assist plasma start-up on tokamak without loop voltage will be very interesting on experiments like ITER to increase the operational margin for a robust breakdown and low-Z impurity burn-through, and also to improve the conditioning techniques with ECRH [7].

\section{ACKNOWLEDGMENTS}

This work was in part supported by the Helmholtz Association and carried out within the framework of the Helmholtz Postdoc Programme.

\section{REFERENCES}

1. V. Erckmann et al., Proc. $20^{\text {th }}$ RFPPC P2.06, Sorrento, 25-25 June 2013, this conference.

2. B. Lloyd et al., Plasma Phys. Control. Fusion 38 (1996)

3. M. Otte et al., Proc. 17th International Stellarator and Heliotron Workshop, Princeton, 12-16 October 2009 (2009).

4. K. Nagasaki et al., Journal of the Korean Physical Society 49 (2006) S18 S24.

5. G. L. Jackson et al., Fusion Sciences and Technology 57 (2010).

6. J. Stober et al., Nucl. Fusion 51083031 (2011).

7. T. Wauters et al., Proc. $20^{\text {th }}$ RFPPC P1.02, Sorrento, 25-25 June 2013, this conference. 